\title{
ARTICLE
}

\section{Hyperprolactinaemia: a guide for psychiatrists ${ }^{\dagger}$}

\author{
Ovais Wadoo, Aadil Jan Shah, Rachael Hall \& Yaasir Mamoojee
}

Ovais Wadoo is a consultant psychiatrist (locum) with Tees, Esk \& Wear Valleys NHS Foundation Trust, at Roseberry Park Hospital, Middlesbrough. Aadil Jan Shah is regional lead consultant at Wirral Ways to Recovery - Change, Grow, Live, in Birkenhead. Rachael Hall is a consultant psychiatrist. Tees, Esk \& Wear Valleys NHS Foundation Trust, at Roseberry Park Hospital, Middlesbrough. Yaasir

Mamoojee is a specialist registrar in diabetes and endocrinology, in the Endocrinology Unit at the Royal Victoria Infirmary, Newcastle Upon Tyne, UK. Correspondence Dr Ovais Wadoo, Tees, Esk \& Wear Valleys NHS Foundation Trust, Roseberry Park Hospital, Marton Road, Middlesbrough TS4 3AF, UK. Email: owadoo@gmail.com

\section{Copyright and usage}

(C) The Royal College of Psychiatrists 2017.

${ }^{\dagger}$ Dr Wadoo and colleagues' e-learning module on this topic is available from the Royal College of Psychiatrists' CPD Online: click on The management of hyperprolactinemia in psychiatric practice or go to http://www. psychiatrycpd.co.uk

\begin{abstract}
SUMMARY
Medications prescribed by psychiatrists are known to elevate serum prolactin levels, but hyperprolactinaemia remains underrecognised, as the adverse effects of an elevated prolactin are mostly not visible. Hyperprolactinaemia can lead to adverse health outcomes, so clinicians need not only to be alert to its symptoms, but to manage the consequences as well. In this article we provide a brief overview of prolactin physiology, regulation and function. We list various factors that can lead to elevated serum prolactin. We discuss the interpretation of blood results and the management of psychotropicinduced hyperprolactinaemia. We include a flow diagram to assist clinicians in decision-making in the clinical management of hyperprolactinaemia.
\end{abstract}

\section{LEARNING OBJECTIVES}

- Understand prolactin physiology and regulation

- Understand hyperprolactinaemia and its causes

- Know the consequences of hyperprolactinaemia and appropriately manage it in clinical practice

\section{DECLARATION OF INTEREST}

None

\section{The hypothalamus and pituitary gland}

The pituitary gland is attached to the hypothalamus by the infundibulum or pituitary stalk; it consists of an anterior and a posterior lobe. The hypothalamus communicates with the anterior pituitary gland by releasing stimulatory and inhibitory hormones into the hypophyseal portal system. These are then transported to the anterior pituitary gland to act on their respective cell receptors. In contrast, the posterior pituitary gland is an extension of the hypothalamus. Hypothalamic neurosecretory cells project directly into the posterior pituitary through the pituitary stalk. Posterior pituitary hormones are released into the systemic circulation via the hypophyseal veins.

The various endocrine cells of the anterior pituitary gland secrete five major hormones. These are: adrenocorticotropic hormone (ACTH), which stimulates the adrenal gland to produce cortisol; thyroid-stimulating hormone (TSH), which acts on the thyroid gland to regulate thyroid hormone synthesis and secretion; growth hormone (GH); gonadotropins, including luteinising hormone $(\mathrm{LH})$ and follicle-stimulating hormone (FSH), which regulate sex hormone production and fertility; and prolactin. The posterior pituitary gland produces two major hormones: vasopressin (also known as antidiuretic hormone, $\mathrm{ADH}$ ) and oxytocin. $\mathrm{ADH}$ is responsible for water and sodium homoeostasis in the body, and oxytocin plays a major role in uterine contraction during labour and the milk 'let-down' response (Wass 2011).

\section{Prolactin}

\section{Source and secretion}

Prolactin is a single-chain polypeptide hormone which is primarily secreted by lactotrophs present in the anterior pituitary gland. It follows a diurnal variation, with serum levels higher at night than during the day. It is secreted in a pulsatile manner - up to 14 peaks a day. The mean pulse amplitude above the preceding nadir is on average about 20$30 \%$ of the upper normal value (Haddad 2004). Females have higher levels than males. Physiological increase in prolactin occurs in the second half of the menstrual cycle, during pregnancy, lactation and nipple stimulation. Transient increase in serum levels occurs during sexual intercourse, exercise and stress. Extra-pituitary sources of prolactin come from the uterus, mammary glands and immune cells. The clinical significance of these sources is less well established.

\section{Regulation}

The release of prolactin from the anterior pituitary gland is tonically inhibited by the hypothalamus through the release of dopamine. The control of prolactin secretion is regulated primarily through dopamine as a prolactin-release inhibiting factor (PIF). Dopamine binds to receptors on lactotrophs, and inhibits both the synthesis and secretion of prolactin (Fig. 1). Other hormones may act as a prolactin-releasing factor (PRF). Examples include thyrotropin-releasing hormone (TRH) and vasoactive intestinal peptide (VIP). 


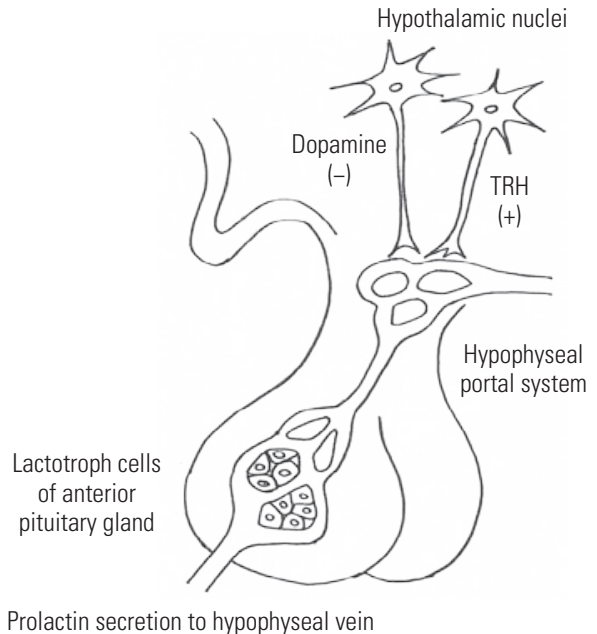

Regulation of prolactin secretion in the hypothalamopituitary system; TRH, thyrotropin-releasing hormone.

\section{Function}

Prolactin's main physiological role is to induce lactation in women. During pregnancy progesterone plays a major role in priming the female breast for lactogenesis (milk production) through stimulation of tubuloalveolar gland development. Prolactin levels gradually rise during pregnancy, peaking at delivery (Tyson 1972). Prolactin then stimulates lactogenesis once the progesterone level abruptly falls after delivery and with removal of the placenta. The let-down response during lactation is mediated by oxytocin, a posterior pituitary hormone, through stimulation of myoepithelial cells in ductal smooth muscles. Prolactin may act as an autocrine or paracrine ${ }^{\mathrm{a}}$ modulator of immune activity.

\section{What is hyperprolactinaemia?}

Hyperprolactinaemia is defined as an elevated serum prolactin level sustained on repeat testing. Measured serum prolactin in humans is considered as almost entirely derived from the pituitary gland. The reference values for a normal prolactin level vary between assays, so the laboratory performing the prolactin assay will provide the reference value alongside the measured one. The upper limit of a normal prolactin level is lower in men than in women, and a prolactin level above $20 \mu \mathrm{g} / \mathrm{L}$ in men and above $25 \mu \mathrm{g} / \mathrm{L}$ in women is generally considered to be elevated. Some laboratories express the value in mIU/L and give different reference values for each gender. Prolactin concentrations expressed in $\mu \mathrm{g} / \mathrm{L}$ can be converted to mIU/L by multiplying by 21.2 (Canadian Society of Clinical Chemists 1992; Melmed 2011) (Box 1).
There are different types of serum prolactin in the circulation. Serum prolactin can be present as monomeric 'little' prolactin (with a mass of 23 $\mathrm{kDa}$ ) or as polymeric complexes that are bound to immunoglobulin $\mathrm{G}$ ( $\operatorname{IgG}$ ) antibodies. These complexes can range from 150 to $170 \mathrm{kDa}$ in mass and are referred to as 'macroprolactin' or 'big big prolactin' (Abel 2006; Kavanagh-Wright 2009). They are slower to be cleared from the circulation by the kidneys. An elevated serum prolactin level can thus be either monomeric prolactin or macroprolactin. Standard laboratory prolactin immunoassays will not differentiate these two types of prolactin, but differentiating between them is clinically relevant. Only monomeric prolactin is biologically active and of clinical significance, whereas macroprolactin is not. The prevalence of macroprolactin accounting for an elevated serum prolactin level has been estimated to range from 10 to $46 \%$, with one retrospective study reporting a $22 \%$ prevalence (Abel 2006). The newer prolactin immunoassays are less sensitive to macroprolactin, hence the lower prevalence rate in most recent studies. The prevalence of macroprolactinaemia is low in the general population and higher in

\section{BOX 1 Serum prolactin levels}

- Prolactin in healthy individuals is considered as almost entirely pituitary prolactin.

- A sustained level of serum prolactin above the upper limit of normal confirms a diagnosis of hyperprolactinaemia as long as the serum sample was obtained without excessive venepuncture stress.

- Although some laboratories adjust their reference values to account for venepuncture stress, in our experience the effect of venepuncture stress on prolactin elevation is highly variable and may reach up to $1500 \mathrm{mIU} / \mathrm{L}(70.75 \mu \mathrm{L})$. Serial measurements of serum prolactin through an indwelling cannula (cannulated prolactin) over a period of time will help diagnose stress-induced hyperprolactinaemia, which shows in decreasing prolactin levels over time. This may not be readily performed in routine psychiatric practice and hence if stress hyperprolactinaemia is suspected referral to an endocrinologist is recommended.

- Given the diurnal variation in serum prolactin levels, some authorities advocate serum sampling at least 1 hour after waking or eating. This may not be clinically practical and we therefore recommend repeated testing to confirm an initial elevated prolactin level.

- The reference value for a normal prolactin level is not standardised and varies between assays; consequently, the laboratory performing the prolactin assay will provide the reference value. a. Autocrine hormones act on the cells that produce them; paracrine hormones act on nearby cells. 
BOX 2 Interpreting the results of prolactin blood tests

- The presence of macroprolactin will result in high measured prolactin levels in the absence of an underlying increase in prolactin secretion

- Check whether the laboratory routinely measures macroprolactin

- If the blood results show high prolactin, request macroprolactin levels

- It is the increased monomeric prolactin that matters

- Routine screening for macroprolactin could eliminate unnecessary diagnostic testing and treatment

the population presenting with high prolactin levels (Sánchez-Eixerés 2001). Whichever assay is used, and irrespective of the prevalence rate, macroprolactin should be excluded in all patients with newly diagnosed hyperprolactinaemia.

Box 2 summarises the key points in interpreting prolactin blood test results.

\section{Physiological and medical causes of hyperprolactinaemia (Wass 2011)}

Of interest to psychiatrists is drug-induced hyperprolactinaemia, but it is important that clinicians are able to rule out non-drug-related causes and that they request appropriate investigations and/or referrals while evaluating the patient. Consultation with an endocrinologist is always advisable if there is any doubt about the underlying cause.

In addition to the physiological factors mentioned in the section on 'Source and secretion', some of the important medical causes are outlined below. The full list of factors that can lead to hyperprolactinaemia is beyond the remit of this article.

\section{Hypothalamic disease}

Destruction of the dopaminergic neuronal cells of the hypothalamus will interrupt the tonic inhibition of the lactotroph cells in the anterior pituitary gland and cause hyperprolactinaemia. Both benign and malignant tumours in the hypothalamus and infiltrative diseases can cause this.

\section{Pituitary stalk interruption}

Dopamine produced by the hypothalamus travels to the anterior pituitary gland through the blood supply of the pituitary stalk. Interruption of the stalk via compressive effects of surrounding large tumours of the pituitary gland or hypothalamus or traumatic section of the stalk will again remove the dopaminergic inhibition to the lactotroph cells and cause hyperprolactinaemia. Tumours compressing the pituitary stalk may also impinge on the optic chiasm and present with bitemporal hemianopia. The patient may complain of loss of vision on the lateral sides of the visual fields.

\section{Pituitary adenoma}

Prolactin-secreting pituitary tumours, also called prolactinomas, cause elevation of prolactin. These tumours vary in size from microadenomas $(<1 \mathrm{~cm}$ in diameter) to macroadenomas $(>1 \mathrm{~cm})$. The degree of hyperprolactinaemia is proportional to the size of the tumour. Non-prolactin-producing tumours of the pituitary gland may also cause a degree of hyperprolactinaemia if they affect the pituitary stalk as previously described. If the optic chiasm is affected, the tumours may present with headaches and visual symptoms.

\section{Hypothyroidism}

Primary hypothyroidism causes a rise in TRH from the hypothalamus, which acts as a prolactinreleasing factor to elevate serum prolactin levels. Patients may complain of weight gain, lethargy, cold intolerance, dry skin, constipation and menstrual irregularities. Adequate replacement of thyroid hormone will normalise TRH levels and subsequently prolactin levels.

\section{Chest-wall injury}

Injuries to the chest wall can elevate prolactin levels via stimulation of neural pathways similar to the suckling response.

\section{Renal failure}

Reduction in prolactin clearance by the kidneys will cause an increase in serum prolactin levels. This is usually detected by a routine blood test for renal function.

\section{Medications associated with hyperprolactinaemia}

Medications are the most common cause of nontumour-related high serum prolactin levels. Various medications can cause a range of elevation in levels (La Torre 2007), but antipsychotic drugs are the most common culprits in hyperprolactinaemia.

The underlying mechanism of prolactin elevation resides in the removal of tonic dopaminergic inhibition from the hypothalamus through $\mathrm{D}_{2}$-receptor blockade on the lactotroph cells (Holt 2011). Such direct inhibition is seen with antipsychotic drugs. Raised prolactin levels can also be caused by inhibition of dopamine synthesis (by drugs such as methyldopa) or of its release from 
dopamine-producing cells. For some other drugs, such as antidepressants, the mechanism is less well understood. The main classes of medication that affect prolactin levels are shown in Table 1.

Not all antipsychotics have this effect. Table 2 illustrates the frequency of hyperprolactinaemia with various commonly prescribed antipsychotic medications. The magnitude of prolactin elevation varies from $17 \mu \mathrm{g} / \mathrm{L}$ with haloperidol to $80 \mu \mathrm{g} / \mathrm{L}$ with risperidone (David 2000). The aetiology of an elevated prolactin level in a patient taking an antipsychotic known to cause hyperprolactinaemia can be confidently established if a normal baseline prolactin level is available before starting the medication (Byerly 2007). If the antipsychotic is the cause of the high prolactin then the level can be expected to normalise following discontinuation of the drug (De Rivera 1976). With an oral daily preparation this may take a few days, but a depot preparation would undoubtedly take longer.

\section{Clinical consequences of hyperprolactinaemia}

Hyperprolactinaemia can have clinically significant health consequences (Perkins 2004). Individuals vary in their sensitivity to the condition and in the symptoms that they develop, owing to variation in the dopamine $\mathrm{D}_{2}$-receptor gene. Thus, elevation in serum prolactin levels may not always translate into clinically evident symptoms, irrespective of the degree of elevation. High prolactin levels may suppress the reproductive axis, leading to reduced gonadotropin levels (FSH and $\mathrm{LH}$ ) and subsequent down-regulation of oestrogen levels in women and testosterone in men, the consequences of which are sexual dysfunction, infertility and bone demineralisation.

\section{Effect on the mammary glands}

Hyperprolactinaemia can cause gynaecomastia and spontaneous galactorrhoea of varying severity. Such galactorrhoea is common in premenopausal women, but it is a rare occurrence in men. It is worth noting that not all patients presenting with galactorrhoea have elevated prolactin levels. In a study of 235 such patients, $46 \%$ had normal prolactin levels (Kleinberg 1977).

\section{Effect on sexual function}

Hyperprolactinaemia can cause sexual dysfunction, although the evidence is more consistent for men than for women. Hypogonadal symptoms include reduced or lost libido, lack of energy, atrophic changes in the urethral and vaginal mucosa, dyspareunia, hot flushes and vaginal dryness in women and erectile dysfunction in
TABLE 1 Effects of various classes of medication on prolactin levels

\begin{tabular}{|c|c|c|}
\hline Class of medication & Frequency of effect & Mechanism of action \\
\hline Antipsychotics & \multicolumn{2}{|l|}{ See Table 2} \\
\hline \multicolumn{2}{|l|}{ Antidepressants (tricyclics) } & \multirow{5}{*}{$\begin{array}{l}\text { Mechanism not well } \\
\text { understood, but an } \\
\text { indirect modulation of } \\
\text { prolactin release by } \\
\text { serotonin has recently } \\
\text { been postulated }\end{array}$} \\
\hline Amitriptyline & + & \\
\hline Clomipramine & +++ & \\
\hline Desipramine & + & \\
\hline Nortriptyline & - & \\
\hline \multicolumn{3}{|l|}{ Antidepressants (SSRIs) } \\
\hline $\begin{array}{l}\text { Citalopram, fluoxetine, } \\
\text { fluvoxamine, paroxetine, } \\
\text { sertraline }\end{array}$ & \multicolumn{2}{|l|}{-} \\
\hline \multicolumn{3}{|l|}{ Antidepressants (other) } \\
\hline $\begin{array}{l}\text { Bupropion, mirtazapine, } \\
\text { trazodone, venlafaxine }\end{array}$ & \multicolumn{2}{|l|}{-} \\
\hline Opiates and cocaine & $\begin{array}{l}\text { Transient increase } \\
\text { in prolactin after } \\
\text { administration }\end{array}$ & $\begin{array}{l}\text { Indirect effect of } \\
\mu \text {-opiate receptor } \\
\text { activation }\end{array}$ \\
\hline \multicolumn{3}{|l|}{ Antihypertensives } \\
\hline Methyldopa & ++ & $\begin{array}{l}\text { Enzymatic blockade } \\
\text { of conversion of } \\
\text { L-dopa to dopamine } \\
\text { and suppression of } \\
\text { dopamine synthesis }\end{array}$ \\
\hline Verapamil & + & $\begin{array}{l}\text { Hypothalamic blockade } \\
\text { of dopamine secretion }\end{array}$ \\
\hline \multicolumn{3}{|c|}{ Gastrointestinal and anti-emetics } \\
\hline Metoclopramide & +++ & $\mathrm{D}_{2}$-receptor blockade \\
\hline Domperidone & +++ & \\
\hline Oestrogens & + & Lactotroph hyperplasia \\
\hline
\end{tabular}

Frequency of increase to abnormal prolactin levels in chronic use: -, none or low in case reports;,$+<25 \%$; ++ , up to $50 \% ;+++,>50 \%$. SSRI, selective serotonin reuptake inhibitor.

Adapted from: Busche et al (2008); Molitch (2008); Coker \& Taylor (2010)

TABLE 2 Effects of various first- and second-generation antipsychotics on prolactin levels

\begin{tabular}{lc}
\hline Antipsychotic & Frequency of effect \\
\hline First-generation antipsychotics (typicals) & ++ \\
Butyrophenones, e.g. haloperidol & ++ to \\
Phenothiazines, e.g. chlorpromazine, fluphenazine, & \\
levomepromazine, perphenazine, prochlorperazine, & ++ \\
trifluoperazine & \\
Thioxanthenes, e.g. flupentixol, zuclopenthixol & ++ \\
Second-generation antipsychotics (atypicals) & - \\
Amisulpride & - \\
Aripiprazole & + \\
Clozapine & ++ \\
Olanzapine & + \\
Paliperidone & + \\
Ouetiapine & +++ \\
Risperidone & - \\
Ziprasidone & +
\end{tabular}

Frequency of increase to abnormal prolactin levels in chronic use: - , none or low in case reports;,$+<25 \%$; ++ , up to $50 \% ;+++,>50 \%$.

Adapted from: Busche et al (2008); Molitch (2008); Coker \& Taylor (2010). 
men (Schlechte 2003). However, some of these symptoms (e.g. low libido, erectile dysfunction and lethargy) may have other causes, including mental illness, psychosocial adversity, chronic illness, malnutrition, excessive physical exercise schedules and non-hyperprolactinaemic sideeffects of medications.

\section{Effect on fertility}

Infertility secondary to hyperprolactinaemia is well recognised in both sexes (Segal 1979). The underlying mechanism is down-regulation of the reproductive axis. Menstrual irregularities range from short luteal phase to amenorrhoea, the occurrence of which is proportional to the degree of hyperprolactinaemia (Corenblum 1976; Fell 2004; Bargiota 2013).

\section{Musculoskeletal effects}

Hypogonadism secondary to hyperprolactinaemia accelerates bone loss in both women and men. This is due to reduced oestrogen and testosterone levels respectively. Psychiatric patients are often started on antipsychotic medications in their late teens or early twenties and, owing to oestrogen deficiency, may not only acquire a reduced peak bone mass but also experience premature acceleration of bone loss. This may be compounded by an increased prevalence of other secondary risk factors for osteoporosis in this group, namely smoking, increased alcohol consumption and poor dietary intake of calcium. They are thus at an increased risk of osteoporosis, and radiological studies demonstrate clinically significant reduction in bone mineral density (Klibanski 1980; Greenspan 1986; Schlechte 1992; Di Somma 1998).

\section{Other consequences specific to women}

In women, hyperprolactinaemia may be associated with hostility, anxiety and depression, although the severity of these symptoms appears to be mild (Sobrinho 1998; Sonino 2007). Some studies suggest an independent risk of suicide (Brugnoli 2012). Although some studies have found an association between hyperprolactinaemia and breast cancers (Tworoger 2007, 2013), meta-analysis has failed to confirm this (Dekkers 2015). Acne and mild hirsutism can develop, due to the relative increase of androgenic compared with oestrogenic activity. Loss of protective effects of oestrogen may affect cardiovascular and cognitive functions.

\section{Clinical guidance when prescribing drugs associated with hyperprolactinaemia}

Why do psychiatrists fail to recognise hyperprolactinaemia? One reason is that the adverse effects of the condition are mostly not visible and thus remain unrecognised. Another is that clinicians do not routinely ask the relevant questions (e.g. about sexual dysfunction and menstrual irregularities), and patients feel embarrassed to introduce the discussion of sideeffects such as libido or erectile dysfunction. We suggest the following key points for clinicians prescribing medications liable to raise prolactin levels:

- carry out pre-treatment screening for hyperprolactinaemia, as it can assist in determining whether or not a patient's subsequent elevated prolactin level is medication-induced (Byerly 2007)

- establish baseline psychosexual function and menstrual history (Walters 2008)

- routinely enquire about sexual dysfunction, breast changes and menstrual function

- if hyperprolactinaemia is present, evaluate with the patient the merits of ongoing use of psychotropic medication and the potential adverse effects of ongoing hyperprolactinaemia

- note that women of reproductive age appear to be at higher risk of hyperprolactinaemia than postmenopausal women (Holt 2011); unsuspected pregnancy should be ruled out in any fertile female presenting with hyperprolactinaemia

- adverse effects, particularly sexual dysfunction, may significantly affect medication adherence, with the potential of discontinuation of treatment and a risk of relapse

- antipsychotics vary in their propensity to cause hyperprolactinaemia (Table 2): the strongest predictors of the condition are the type and dose of the antipsychotic prescribed (Inder 2011).

\section{Management of hyperprolactinaemia}

The management of hyperprolactinaemia initially requires a thorough clinical assessment of the patient and appropriate investigations to reveal the aetiology (Fig. 2). Subsequent management is dependent on the cause of the raised prolactin. It is widely held that, in the absence of symptoms or osteoporosis, there is no need to 'treat' druginduced hyperprolactinaemia. It is important to note that the presence and severity of clinical symptoms, and not a rise in prolactin level alone, should dictate treatment strategy (Byerly 2007). With successful management, restoration of menstrual cycles in women will lead to improved fertility. Prompt consideration should be given to contraceptive advice if pregnancy is not desired, or to pre-pregnancy counselling in the case of the contrary. 


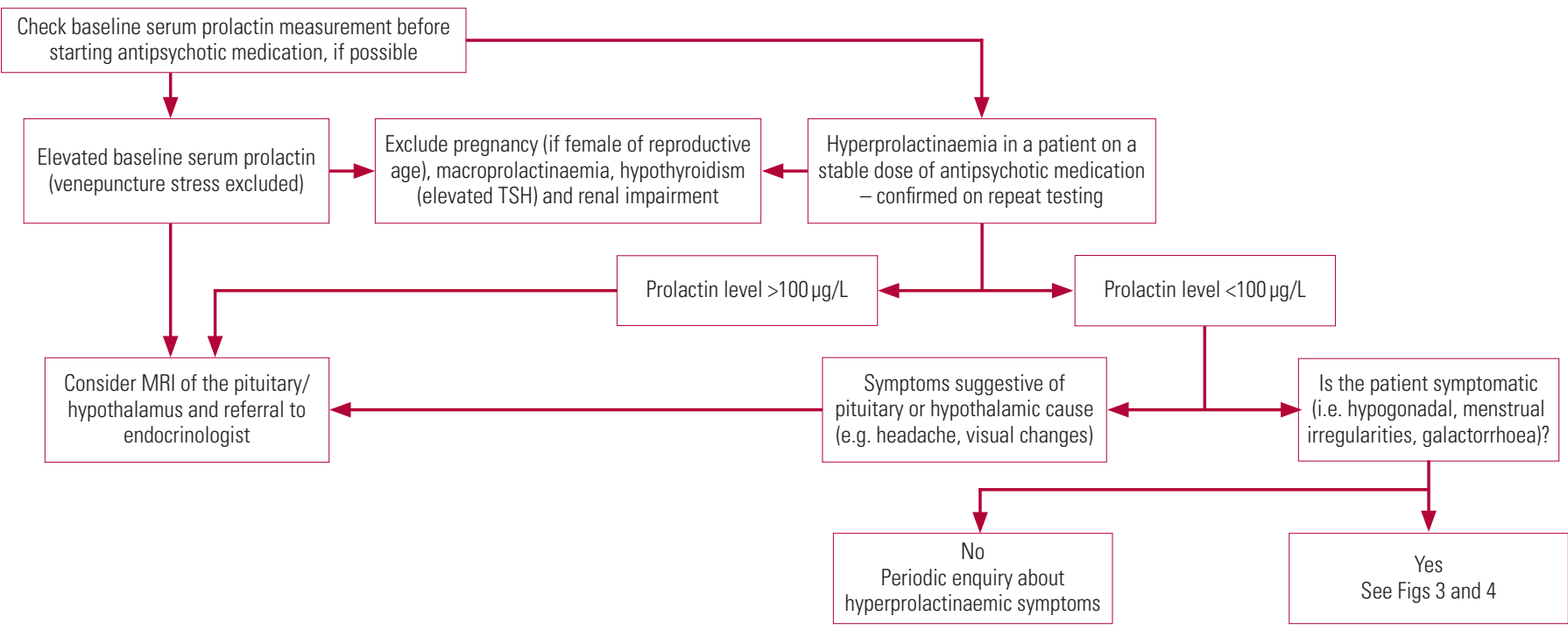

Key steps in identifying and managing antipsychotic-associated hyperprolactinaemia. MRI, magnetic resonance imaging; TSH, thyroid-stimulating hormone.

We suggest the following stepwise approach to managing hyperprolactinaemia associated with antipsychotics (summarised in Figs 3 and 4). Its management in patients desiring fertility and in adolescents is beyond the scope of this article.

\section{Stepwise management of symptomatic antipsychotic-induced hyperprolactinaemia}

\section{Dose reduction}

Reduce the dose of the offending antipsychotic with a potential plan to discontinue it (Holt 2011). For most patients, discontinuation will not be possible, given the risk of deterioration in mental health, and the decision should be made on the basis of collaborative discussion with the patient regarding the risks versus the benefits. It is not recommended to discontinue antipsychotic therapy in asymptomatic patients (Melmed 2011).

\section{Substitution}

Switch the patient to a prolactin-sparing agent (Box 3). The risks and benefits of changing medications should be adequately weighed with the patient and a shared decision-making process undertaken.

\section{Adjunctive aripiprazole}

Add another antipsychotic with dopamine agonist properties - commonly aripiprazole, which is a partial dopamine agonist. This agent may normalise prolactin levels when used on its own or when added to a different antipsychotic. A dose of $10-30 \mathrm{mg}$ once daily is required (Shim 2007; Byerly 2009; Kotorki 2010; Kelly 2013). Although aripiprazole's effect on prolactin is dose-related, low doses can still be effective and therefore starting low and titrating according to response

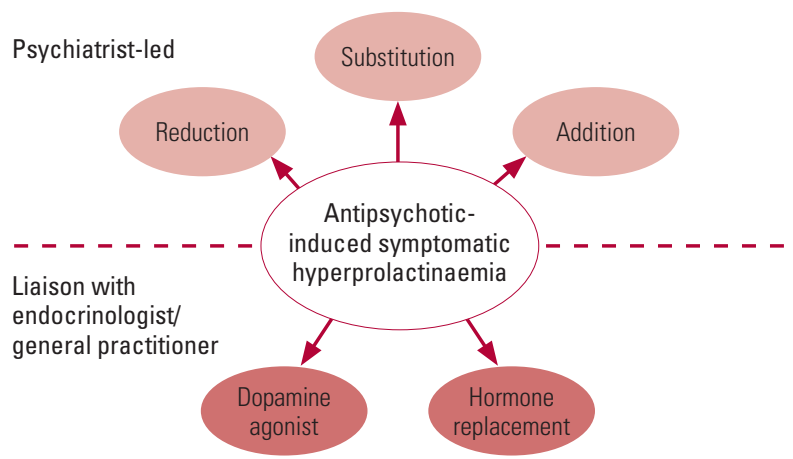

FIG 3 Outline of management strategies for antipsychotic-induced hyperprolactinaemia.

is advised. Doses as high as $30 \mathrm{mg}$ are rarely necessary. The aim is not necessarily to normalise prolactin levels - rather to achieve resolution of symptoms, which may occur before prolactin is normalised. Aripiprazole does not always lower prolactin when added to antipsychotics - there is literature evidence that it is not as effective at lowering levels when the prolactin-raising antipsychotic is a benzamide such as amisulpride.

\section{Adjunctive bromocriptine or cabergoline}

Add bromocriptine or cabergoline (which are also dopamine agonists). This is the least favoured

BOX 3 Prolactin-sparing antipsychotics

\begin{tabular}{ll}
\hline - Aripiprazole & • Olanzapine \\
- Asenapine & - Ouetiapine \\
- Clozapine & • Ziprasidone \\
- Lurasidone &
\end{tabular}


approach as these agents may counteract the beneficial psychiatric effects of antipsychotics and heighten the risk of relapse (Chang 2008). This strategy should only be undertaken when other options are not possible or are ineffective. If this strategy is undertaken, a cautious approach is mandatory, with input from an endocrinologist.

\section{Hormone replacement}

If the offending antipsychotic cannot be discontinued or substituted and an adjunctive dopamine agonist does not normalise prolactin levels, oestrogen or testosterone therapy should be considered if the patient has hypogonadal symptoms or low bone mass (Inder 2011). Hormonal therapy to treat hypogonadism in both men and women will restore general well-being by improving libido, energy levels and sexual function. In premenopausal women, hormone replacement therapy (HRT) with oestrogen and progestogens (if the patient has an intact uterus) will normalise menstrual irregularities if fertility is not desired. In men, testosterone therapy will treat hypogonadal symptoms. Liaison with an endocrinologist or the general practitioner will be required for hormone replacement.

\section{Osteopenia and osteoporosis}

Bone mineral density should be assessed in all patients on a prolonged course of antipsychotics who have been hypogonadal for a long time.

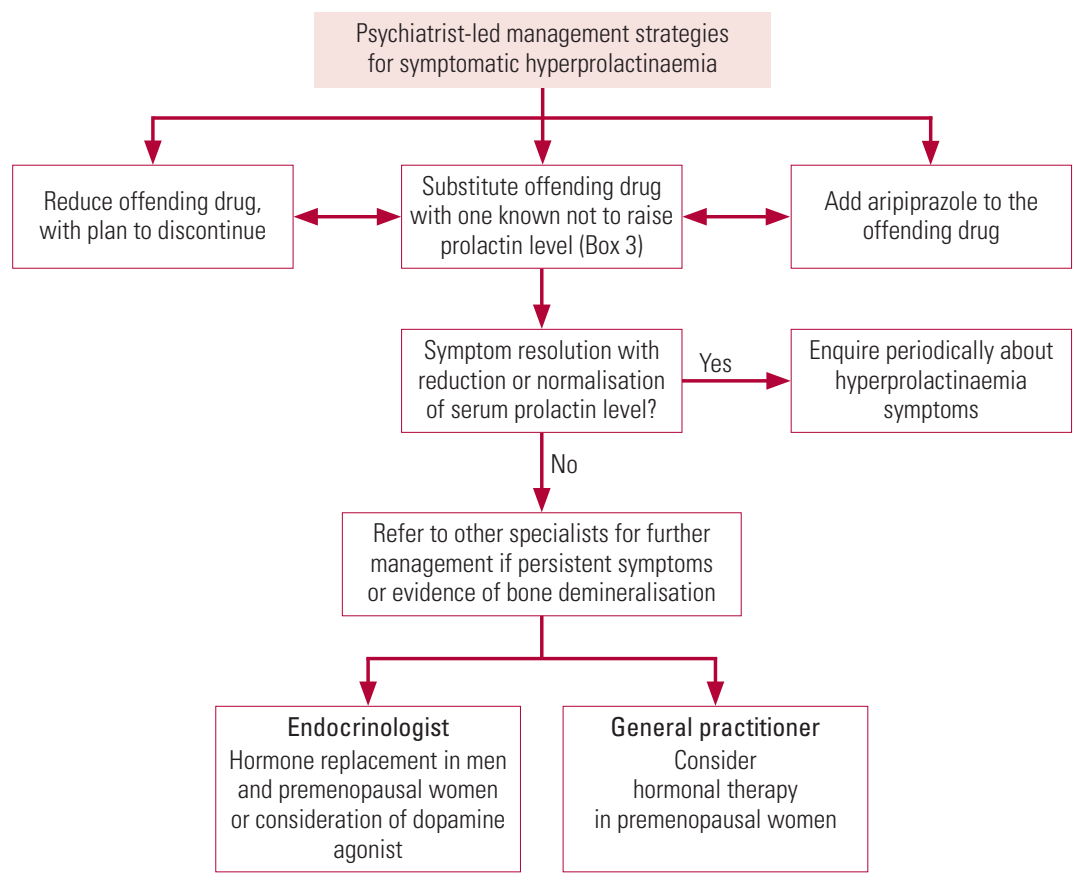

FIG 4

Flowchart of psychiatrist-led strategies for managing antipsychotic-induced hyperprolactinaemia.
Although suppression of the pituitaryhypothalamic-gonadal axis occurs concurrently with hyperprolactinaemia, bone demineralisation is a slower process and may take up to 5 years to become evident on bone densitometry.

If osteopenia or osteoporosis is present, then further monitoring and management of bone mineral density will be needed after starting hormonal therapy and the assistance of a rheumatologist may be required.

Patients started on antipsychotics should be advised on a general healthy lifestyle throughout their treatment to prevent osteoporosis. Smoking, increased alcohol intake, lack of physical exercise and restricted diet are modifiable risk factors for osteoporosis.

\section{Monitoring of prolactin levels}

As mentioned earlier, pre-treatment screening for hyperprolactinaemia can help in determining whether or not a subsequent elevated prolactin level is due to medication. This is reflected in the National Institute for Health and Care Excellence (NICE; 2014) clinical guideline and in the Maudsley Prescribing Guidelines (MPG) (Taylor 2015). These guidelines are less clear on repeat testing: NICE does not mention it; the MPG recommend repeat tests at 6 months and then yearly for all patients on antipsychotics (p. 46), with testing earlier if questions about prolactin-related symptoms (sexual dysfunction, amenorrhoea, etc.) at 3 months raise suspicions of hyperprolactinaemia (p. 133). Prolactin may rise within hours of antipsychotics entering the system. The prolactin level should be measured once the patient is on a stable dose, but further monitoring is not indicated in someone with raised prolactin but no symptoms. Enquiring about hyperprolactinaemic symptoms periodically would be advisable, and using the reporting of symptoms as a trigger to check prolactin levels is a reasonable strategy.

It is important to note that hyperprolactinaemia with suppression of the hypothalamopituitarygonadal (HPG) axis is clinically evident in amenorrhoeic women of childbearing age but may be less evident in men. The perception of hypogonadal symptoms in men varies widely, from our anecdotal evidence. Although using symptoms as a trigger to check for raised prolactin levels may be a reasonable, simple and resource-sparing strategy, there may be a proportion of asymptomatic patients (especially men) with medication-induced HPG suppression who will be missed. Whether this translates into poor bone health over time and suboptimal well-being is less clear. Some clinicians 
do test prolactin levels 3 or 6 months after initiation of antipsychotics and annually thereafter and do check testosterone levels in male patients with medication-induced hyperprolactinaemia.

\section{References}

Abel A, Kimberly IR, Robert AV (2006) Incidence and clinical significance of elevated macroprolactin levels in patients with hyperprolactinemia. Endocrine Practice, 12: 275-80.

Bargiota SI, Bonotis KS, Messinis IE, et al (2013) The effects of antipsychotics on prolactin levels and women's menstruation. Schizophrenia Research and Treatment, 24 Dec: doi 10.1155/2013/502697 [Epub].

Brugnoli R, Novick D, Maria Haro J, et al (2012) Risk factors for suicide behaviors in the observational schizophrenia outpatient health outcomes (SOHO) study. BMC Psychiatry, 12: 83.

Busche C, Yeomans D, Floyd T, et al (2008) Categorical prevalence and severity of hyperprolactinaemia in two UK cohorts of patients with severe mental illness during treatment with antipsychotics. Journal of Psychopharmacology, 22: 56-62.

Byerly M, Suppes T, Tran QV, et al (2007) Clinical implications of antipsychotic-induced hyperprolactinemia in patients with schizophrenia spectrum or bipolar spectrum disorders: recent developments and current perspectives. Journal of Clinical Psychopharmacology, 27: 639-61.

Byerly MJ, Marcus RN, Tran OV et al (2009) Effects of aripiprazole on prolactin levels in subjects with schizophrenia during cross-titration with risperidone or olanzapine: analysis of a randomized, open-label study. Schizophrenia Research, 107: 218-22.

Canadian Society of Clinical Chemists (1992) Canadian Society of Clinical Chemists position paper: standardization of selected polypeptide hormone measurements. Clinical Biochemistry, 25: 415-24.

Chang SC, Chen C-H, Lu M-L (2008) Cabergoline-induced psychotic exacerbation in schizophrenic patients. General Hospital Psychiatry, 30: 378-80.

Coker F, Taylor D (2010) Antidepressant-induced hyperprolactinaemia incidence, mechanisms and management. CNS Drugs, 24: 563-74.

Corenblum B, Pairaudeau N, Shewchuk AB (1976) Prolactin hypersecretion and short luteal phase defects. Obstetrics and Gynecology, 47: 486-8.

David SR, Taylor CC, Kinon BJ, et al (2000) The effects of olanzapine, risperidone, and haloperidol on plasma prolactin levels in patients with schizophrenia. Clinical Therapeutics, 22: 1085-96.

Dekkers OM, Ehrenstein V, Bengtsen M, et al (2015) Breast cancer risk in hyperprolactinemia patients: a population-based cohort study and meta-analysis of the literature. European Journal of Endocrinology, 173 : 269-73

De Rivera JL, Lal S, Ettigi P, et al (1976) Effect of acute and chronic neuroleptic therapy on serum prolactin levels in men and women of different age groups. Clinical Endocrinology, 5: 273-85.

Di Somma C, Colao A, Di Sarno A, et al (1998) Bone marker and bone density responses to dopamine agonist therapy in hyperprolactinemic males. Journal of Clinical Endocrinology and Metabolism, 83: 807-13.

Fell MJ, Neill JC, Marshall KM (2004) Effects of the classical antipsychotic haloperidol and atypical antipsychotic risperidone on weight gain, the oestrous cycle and uterine weight in female rats. European Neuropsychopharmacology, 14: 385-92.

Greenspan SL, Neer RM, Ridgway EC, et al (1986) Osteoporosis in men with hypoprolactinemic hypogonadism. Annals of Internal Medicine, 104: $777-82$

Haddad PM, Wieck A (2004) Antipsychotic-induced hyperprolactinaemia mechanisms, clinical features and management. Drugs, 64: 2291-314.

Holt RIG, Peveler RC (2011) Antipsychotics and hyperprolactinaemia: mechanisms, consequences and management. Clinical Endocrinology, 74: $141-7$

Inder WJ, Castle D (2011) Antipsychotic-induced hyperprolactinaemia. Australian and New Zealand Journal of Psychiatry, 45: 830-7.
Kavanagh-Wright L, Smith TP, Gibney J, et al (2009) Characterization of macroprolactin and assessment of markers of autoimmunity in macroprolactinaemic patients. Clinical Endocrinology, 70: 599-605.

Kelly DL, Wehring HJ, Earl AK, et al (2013) Treating symptomatic hyperprolactinemia in women with schizophrenia: presentation of the ongoing DAAMSEL clinical trial (Dopamine partial Agonist, Aripiprazole, for the Management of Symptomatic ELevated prolactin). BMC Psychiatry, 13: 214.

Kleinberg DL, Noel GL, Frantz AG (1977) Galactorrhea: a study of 235 cases, including 48 with pituitary tumors. New England Journal of Medicine, 296: 589-600.

Klibanski, Neer RM, Beitins IZ, et al (1980) Decreased bone density in hyperprolactinemic women. New England Journal of Medicine, 303 : $1511-4$.

Kotorki P, Pelka P, Leotsakou C, et al (2010) Reversal of symptomatic antipsychotic-induced hyperprolactinemia with addition of aripiprazole. Annals of General Psychiatry, 9 (suppl 1): S164.

La Torre D, Alberto F (2007) Pharmacological causes of hyperprolactinemia. Therapeutics and Clinical Risk Management, 3: 929-51.

Melmed S, Casanueva FF, Hoffman AR, et al (2011) Diagnosis and treatment of hyperprolactinemia: an Endocrine Society clinical practice guideline. Journal of Clinical Endocrinology \& Metabolism, 96: 273-88.

Molitch ME (2008) Drugs and prolactin. Pituitary, 11: 209-18.

National Institute for Health and Care Excellence (2014) Psychosis and Schizophrenia in Adults: Prevention and Management (Clinical Guideline CG178). NICE.

Perkins D0 (2004) Antipsychotic induced hyperprolactinemia: pathophysiology and clinical consequences. Advanced Studies in Medicine, 4(10F): S982-6.

Sánchez-Eixerés MR, Mauri M, Alfayate R, et al (2001) Prevalence of macroprolactin detected by Elecsys 2010. Hormone Research, 56: 87-92.

Schlechte J, Walkner L, Kathol M (1992) A longitudinal analysis of premenopausal bone loss in healthy women and women with hyperprolactinemia. Journal of Clinical Endocrinology and Metabolism, 75: 698-703.

Schlechte JA (2003) Prolactinoma. New England Journal of Medicine, 349: 2035-41.

Segal S, Yaffe H, Laufer N, et al (1979) Male hyperprolactinemia: effects on fertility. Fertility and Sterility, 32: 556-61.

Shim JC, Shin JG, Kelly DL, et al (2007) Adjunctive treatment with a dopamine partial agonist, aripiprazole, for antipsychotic-induced hyperprolactinemia: a placebo-controlled trial. American Journal of Psychiatry, 164: 1404-10

Sobrinho LG (1998) Emotional aspects of hyperprolactinemia. Psychotherapy and Psychosomatics, 67: 133-9.

Sonino N, Tomba E, Fava GA (2007) Psychosocial approach to endocrine disease. Advances in Psychosomatic Medicine, 28: 21-33.

Taylor D, Paton C, Kapur S (2015) The Maudsley Prescribing Guidelines in Psychiatry (12th edn). Wiley-Blackwell.

Tworoger SS, Eliassen AH, Sluss P, et al (2007) A prospective study of plasma prolactin concentrations and risk of premenopausal and postmenopausal breast cancer. Journal of Clinical Oncology, 25: 1482-8.

Tworoger SS, Eliassen AH, Zhang $X$, et al (2013) A 20-year prospective study of plasma prolactin as a risk marker of breast cancer development. Cancer Research, 73: 4810-9.

Tyson JE, Hwang P, Guyda H, et al (1972) Studies of prolactin secretion in human pregnancy. American Journal of Obstetrics \& Gynecology, 113(1): 14-20.

Walters J, Jones I (2008) Clinical questions and uncertainty: prolactin measurement in patients with schizophrenia and bipolar disorder. Journal of Psychopharmacology, 22 (suppl 2): 82-9.

Wass J, Shalet S, Gale E, et al (2011) Oxford Textbook of Endocrinology and Diabetes (2nd edn). Oxford University Press.

\section{MCQ answers \\ 1 c 2 c 3 e 4 a 5 b}




\section{MCOs}

Select the single best option for each question stem

1 Which of the following hormones is not secreted by the anterior pituitary gland?

a Prolactin

b Thyroid-stimulating hormone (TSH)

c Vasopressin

d Adrenocorticotropic hormone (ACTH)

e Growth hormone (GH).

\section{Which statement regarding prolactin is} correct?

a Prolactin is a polypeptide hormone produced by the posterior pituitary gland and is responsible for lactogenesis (milk production)

b Prolactin secretion is stimulated by dopamine produced by the hypothalamus

c Prolactin induces lactogenesis in the primed female breast and its release from the anterior pituitary gland is inhibited by dopamine produced by the hypothalamus d Prolactin release is inhibited by thyrotropinreleasing hormone (TRH) produced by the hypothalamus

e Prolactin is produced by the anterior pituitary gland and has no physiological role in humans.

3 Which of the following statements is wrong?

a In psychiatric practice, drug-induced hyperprolactinaemia is most likely secondary to antipsychotic medications

b Although first-generation antipsychotics can cause marked elevation in prolactin levels, not all second-generation antipsychotics have this effect

c The degree of prolactin elevation is not usually more than $100 \mu \mathrm{g} / \mathrm{L}$

d Consideration should be given to other pathological causes of hyperprolactinaemia in patients on antipsychotics with prolactin levels above the expected range

e Aripiprazole has only a dopamine antagonist effect.
4 Which of the following statements regarding an elevated prolactin level is true?

a True hyperprolactinaemia is defined as raised monomeric prolactin levels sustained on repeat testing

b Elevated prolactin in the circulation is always monomeric in nature

c Macroprolactinaemia need not be considered in a patient with raised prolactin levels

d Prolactin levels are not affected by pregnancy, breastfeeding, stress and eating

e The reference values for a normal prolactin are standardised and do not vary between assays.

5 Which of the following is not a prolactinsparing antipsychotic?

a Clozapine

b Amisulpride

c Olanzapine

d Quetiapine

e Aripiprazole. 